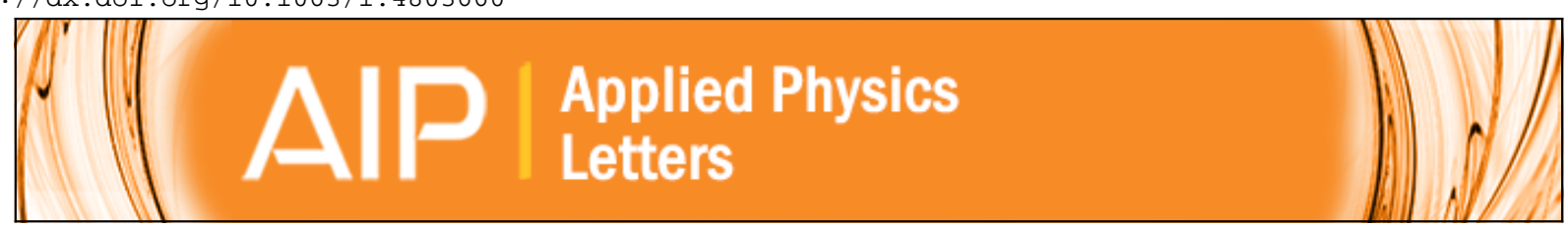

Piezomagnetic strain-dependent non-linear magnetoelectric response enhancement by flux concentration effect

Ying Shen, Junqi Gao, Yaojin Wang, Peter Finkel, Jiefang Li, and D. Viehland

Citation: Applied Physics Letters 102, 172904 (2013); doi: 10.1063/1.4803660

View online: http://dx.doi.org/10.1063/1.4803660

View Table of Contents: http://scitation.aip.org/content/aip/journal/apl/102/17?ver=pdfcov

Published by the AIP Publishing

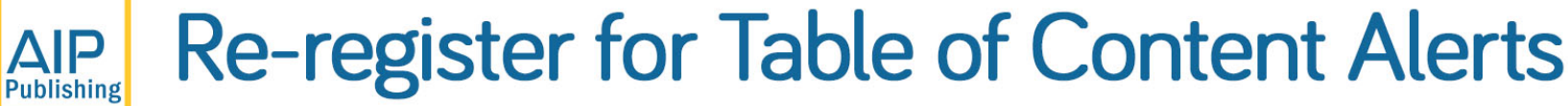

\section{Create a profile. 国 Sign up today!}

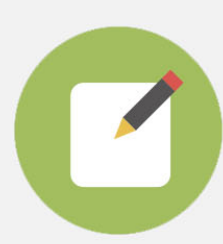




\title{
Piezomagnetic strain-dependent non-linear magnetoelectric response enhancement by flux concentration effect
}

\author{
Ying Shen, ${ }^{1}$ Junqi Gao, ${ }^{1}$ Yaojin Wang, ${ }^{1}$ Peter Finkel, ${ }^{2}$ Jiefang $\mathrm{Li},{ }^{1}$ and D. Viehland ${ }^{1}$ \\ ${ }^{1}$ Department of Materials Science and Engineering, Virginia Tech, Blacksburg, Virginia 24061, USA \\ ${ }^{2}$ Naval Undersea Warfare Center, Newport, Rhode Island 02840, USA
}

(Received 11 March 2013; accepted 14 April 2013; published online 30 April 2013)

\begin{abstract}
The non-linear magnetoelectric (ME) effect of Metglas/ $\mathrm{Pb}\left(\mathrm{Mg}_{1 / 3} \mathrm{Nb}_{2 / 3}\right) \mathrm{O}_{3}-\mathrm{PbTiO}_{3}$ heterostructures has been studied. Such effect holds promise for modulation mode ME sensor applications that require no dc bias. The non-linear ME coefficient was found to be highly dependent on the derivative of the piezomagnetic strain coefficient, which could be increased by increasing the Metglas length due to magnetic flux concentration. The non-linear ME coefficient was equal to $9.5 \mathrm{~V} /\left(\mathrm{cm}-\mathrm{Oe}^{2}\right)$ at $H_{\mathrm{dc}}=-1 \mathrm{Oe}$ and $7.5 \mathrm{~V} /\left(\mathrm{cm}-\mathrm{Oe}^{2}\right)$ at $H_{\mathrm{dc}}=0$ Oe for a structure with $10 \mathrm{~cm}$ long Metglas foils. (C) 2013 AIP Publishing LLC. [http://dx.doi.org/10.1063/1.4803660]
\end{abstract}

Magnetoelectric (ME) effect is an electric output in response to an applied magnetic field, which is mediated by a mechanical-coupling between the magnetostrictive and piezoelectric phases. ${ }^{1,2}$ In a heterostructures configuration where the two phases are engineered with close interface contact, a giant electric response with magnetic field has been found which is described as ME voltage (or charge) coefficient $\alpha^{M E}$. 3,4 The achievement of giant values of $\alpha^{M E}$ in ME heterostructures operated in a passive mode is ready for technological ME device applications. ${ }^{5}$ One promising application is to develop highly sensitive and low-power consuming magnetometers that operate at room temperature and have sensitivity levels at low frequencies approaching that of optically pumped magnetometers. ${ }^{6-9}$ The piezomagnetic effect is linear only when the magnetic field and the stress in the magnetostrictive phase vary within a limited range that is set by a DC magnetic bias $\left(H_{\mathrm{dc}}\right)$ and/or a mechanical prestress. Previous investigations have focused on maximizing $\alpha^{M E}$ near an optimized $H_{\mathrm{dc}}$. Values as high as $\alpha^{M E}$ $=61 \mathrm{~V} / \mathrm{cm} \mathrm{Oe}$ at $f=1 \mathrm{~Hz}$ have been achieved which have low equivalent magnetic noise levels of $6 \mathrm{pT} / \mathrm{rtHz}^{10}$ However, low-frequency vibration and acoustic noises introduced by the piezoelectric effect can significantly raise the noise floor.

A non-linearity in $\alpha^{M E}$ is known which is due to a corresponding non-linearity in the magnetostriction of the Metglas layers under a given $H_{\mathrm{dc}} \cdot{ }^{11}$ Recently, a modulation mode sensor which used a 2nd order ME nonlinearity was reported that transferred low-frequency target signals to higher frequencies. ${ }^{12}$ In this method, an exciting carrier signal provided an effective means to reduce $1 / f$ noise, to increase the signal-to-noise ratio (SNR), and to mitigate vibrational noise that is compared to a conventional passive mode scheme. ${ }^{13}$ The effectiveness of this frequency transfer ability was proportional to the nonlinear $\mathrm{ME}$ coefficient $\alpha_{\text {nonlin. }}^{\mathrm{ME}} \quad$ Recently, for Metglas/Pb( $\left.\mathrm{Mg}_{1 / 3} \mathrm{Nb}_{2 / 3}\right) \mathrm{O}_{3}-\mathrm{PbTiO}_{3}$ laminates, $\alpha_{\text {nonlin }}^{\mathrm{ME}}$ was shown to have an optimum value near $H_{\mathrm{dc}}=0 .{ }^{12}$ The lack of the need for $H_{\mathrm{dc}}$ makes the modulation approach promising. Accordingly, there is a need to understand what causes $\alpha_{\text {nonlin }}^{\mathrm{ME}}$ to vary with $H_{\mathrm{dc}}$. The goal is to find a way to improve $\alpha_{\text {nonlin }}^{\mathrm{ME}}$, especially at $H_{\mathrm{dc}}=0$, which would be enabled for the modulation method to reduce noise by frequency shifting.

Push-pull tri-layer ME laminates were fabricated by bonding two layers of Metglas on a core $\mathrm{Pb}\left(\mathrm{Mg}_{1 / 3} \mathrm{Nb}_{2 / 3}\right) \mathrm{O}_{3}-$ $\mathrm{PbTiO}_{3}$ (PMN-PT) fiber layer. ${ }^{14}$ The PMN-PT (Ceracomp, Korea) fibers had a thickness of $200 \mu \mathrm{m}$, a length of $4 \mathrm{~cm}$, and a width of $1 \mathrm{~cm}$. Both top and bottom surfaces of the core composites were fixed to Kapton interdigitated (ID) copper electrode layers (Smart materials, USA) using an epoxy resin (Stycast 1264, USA). The copper electrodes had $500 \mu \mathrm{m}$ wide digits spaced at a $1.2 \mathrm{~mm}$ (center-to-center) distance, enabling the push-pull configuration. The Metglas foils (Vacuumscheltze $\mathrm{GmbH} \& \mathrm{Co}$. KG, Germany) were $20 \mu \mathrm{m}$ thick and were tailored to a length of $8 \mathrm{~cm}$ and a width of $1 \mathrm{~cm} .{ }^{14}$ Two tri-layer Metglas foils (epoxied to each other) of such dimensions were symmetrically attached on both sides of the core composite using an epoxy resin (West system 206, USA). To minimize the epoxy thickness in between the layers, the laminates were then pressed by a vacuum bag method and cured for more than $24 \mathrm{~h}$ at room temperature.

A 0.074 Oe exciting signal of $H_{\mathrm{ac}}{ }^{*} \sin \left(2 \pi f_{0}\right) \mathrm{t}\left(f_{0}=1 \mathrm{kHz}\right)$ was applied to a coil which wound around the laminate along its longitudinal axis that was driven by a dynamic signal analyzer (SR-785, Stanford Research Systems, USA), as illustrated in Figure 1. A small AC magnetic signal of $180 \mathrm{nT}$ was imposed as an incident field $H_{\text {inc }}{ }^{*} \sin \left(2 \pi f_{1}\right) t\left(f_{1}=1 \mathrm{~Hz}\right)$ by a Helmholtz coil, which surrounded the laminate and was driven by a signal generator. An induced ME modulation output signal of $H_{i n c} H_{a c} \cos \left[2 \pi\left(f_{0} \pm f_{1}\right) t\right]$ was measured by the SR-785. The value of $\alpha_{\text {nonlin }}^{\mathrm{ME}}$ as a function of $H_{\mathrm{dc}}$ is shown in Figure 2(a), which can be defined as ${ }^{12}$

$$
\alpha_{\text {nonlin }}^{\mathrm{ME}}=\frac{\mathrm{E}}{\mathrm{H}_{\mathrm{inc}} \times \mathrm{H}_{\mathrm{ac}}}\left(\frac{\mathrm{V}}{\mathrm{cm}-\mathrm{Oe}^{2}}\right) \text {, }
$$

where $H_{\text {inc }}$ is an incident field (to be measured) of a low characteristic frequency $f_{1}, H_{\mathrm{ac}}$ is the driving or modulation high-frequency field at $f_{0}$, and $E$ is the ME voltage output of a cross-modulation frequency at $f_{0} \pm f_{1}$. 


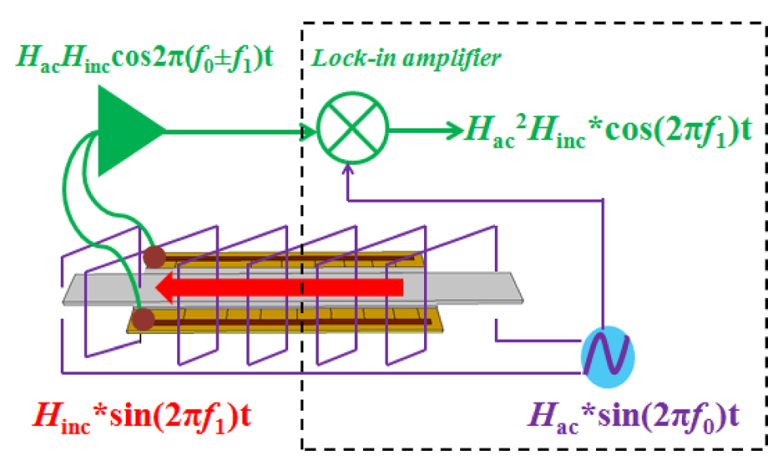

FIG. 1. Schematic illustration of the ME-composite-based modulation. Carrier signal $H_{\mathrm{ac}}{ }^{*} \sin \left(2 \pi f_{0}\right) \mathrm{t}$ was applied by a driving coil which was wound around the laminate; incident field $H_{\text {inc }}{ }^{*} \sin \left(2 \pi f_{1}\right) \mathrm{t}$ was applied through a Helmholtz coil surrounded the ME laminate. The induced modulation signal is $H_{\text {inc }} H_{a c} \cos \left[2 \pi\left(f_{0} \pm f_{1}\right) t\right]$, which can be multiplied with the driving signal $H_{a c} \sin \left(2 \pi f_{0} t\right)$ again via a lock-in amplifier circuits to recover the original incident signal $f_{1}$ as $H_{i n c} H_{a c}^{2} \cos \left(2 \pi f_{1}\right) t$.

In Figure 2(a), the value of $\alpha_{\text {nonlin }}^{\mathrm{ME}}$ can be seen to have multiple peaks with increasing $H_{\mathrm{dc}}$. A value of $4.8 \mathrm{~V} /\left(\mathrm{cm}-\mathrm{Oe}^{2}\right)$ was found at $H_{\mathrm{dc}}=0 \mathrm{Oe}$, which increased to a first maximum near $H_{\mathrm{dc}}= \pm 2 \mathrm{Oe}$ of about $6.5 \mathrm{~V} /\left(\mathrm{cm}-\mathrm{Oe}^{2}\right)$ and subsequently decreased with increasing $H_{\mathrm{dc}}$ until $H_{\mathrm{dc}}= \pm 9$ Oe. With further increase of $H_{\mathrm{dc}}$, the value of $\alpha_{\text {nonlin }}^{\mathrm{ME}}$ increased to a 2 nd peak of $\alpha_{\text {nonlin }}^{M E}=7.5 \mathrm{~V} /\left(\mathrm{cm}-\mathrm{Oe}^{2}\right)$ at $H_{\mathrm{dc}}= \pm 13 \mathrm{Oe}$ and then decreased rapidly approaching a value of zero for $H_{\mathrm{dc}}> \pm 20 \mathrm{Oe}$. Figure 2(b) shows the magnetostriction $\lambda$ and the effective piezomagnetic coefficient $d_{33}^{m}$ (or $\partial \lambda / \partial H)$ as a function of $H_{\mathrm{dc}}$. The value of $\alpha^{M E}$ was strongly dependent on $d_{33}^{m}$, as can be seen in Figure 2(c). The value of $\alpha_{\text {nonlin }}^{M E}$ was also found to be dependent on $\partial d_{33}^{m} / \partial H$. Comparison of Figs. 2(a) and 2(d) will reveal that as $\partial d_{33}^{m} / \partial H$ approached a maximum value of $0.85 \times 10^{-6} \mathrm{Oe}^{-2}$ near $H_{\mathrm{dc}}=0$ Oe the value of $\alpha_{\text {nonlin }}^{\mathrm{ME}}$ was near its 1 st peak, and when $\partial d_{33}^{m} / \partial H$ was at its 2 nd peak near $H_{\mathrm{dc}}= \pm 13$ Oe the $\alpha_{\text {nonlin }}^{M E}$ was also at its 2 nd peak. Furthermore, when $\alpha_{\text {nonlin }}^{M E}$ reached a minimum at $H_{\mathrm{dc}}= \pm 9 \mathrm{Oe}$, the value of $\partial d_{33}^{m} / \partial H$ was also at its minimum.

Next, the relationship between $\alpha_{n o n l i n}^{M E}$ and $d_{33}^{m}$ was analyzed. The ME voltage $V(\mathrm{t})$ can be written in a Taylor expansion series as ${ }^{15}$

$$
V(t)=\alpha_{0}+\alpha_{1} H(t)+\alpha_{2} H^{2}(t)+\alpha_{3} H^{3}(t)+\cdots,
$$

where $\alpha_{\mathrm{i}}(i=0,1,2 \ldots)$ is the Taylor expansion coefficient of $i$ th order. The first order coefficient $\alpha_{1}=\frac{d V}{d H}$ equals $\alpha^{\mathrm{ME}}$. When the laminate is excited by $H_{\mathrm{ac}} * \sin \left(2 \pi f_{0}\right) \mathrm{t}$ with a parallel superimposed incident field $H_{\text {inc }}{ }^{*} \sin \left(2 \pi f_{1}\right)$ t, an effective
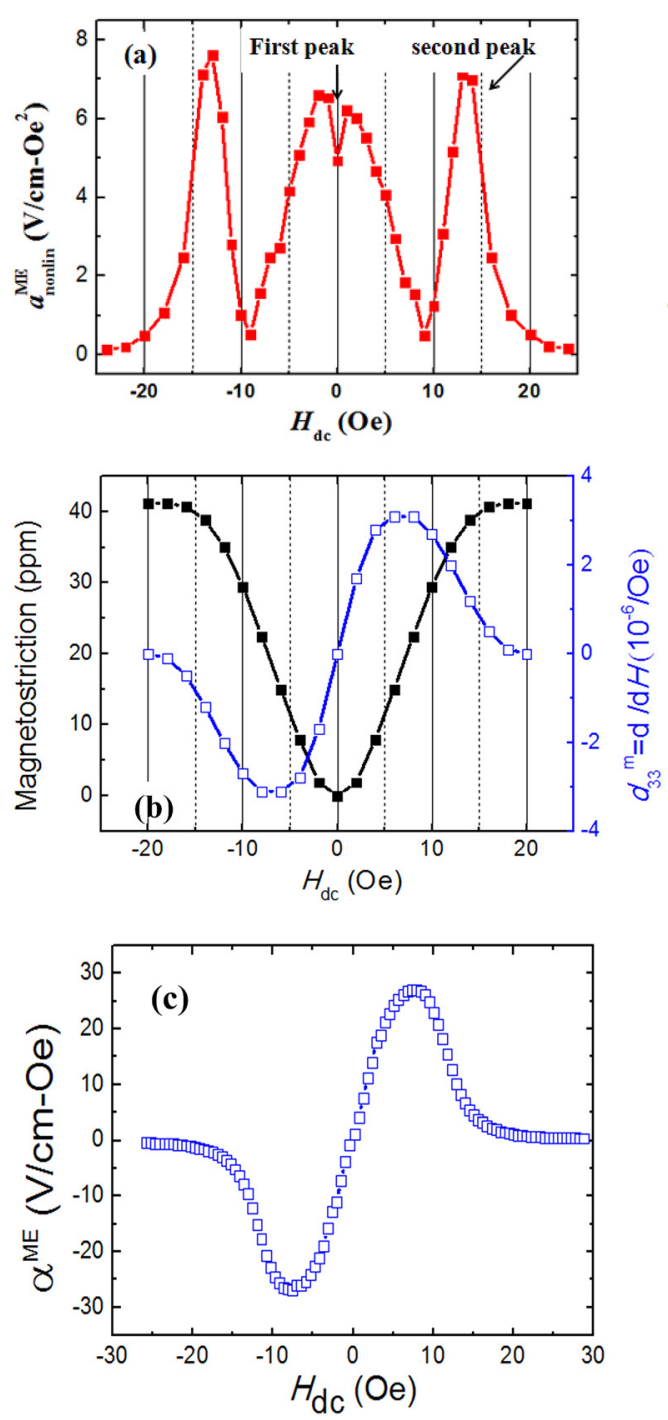
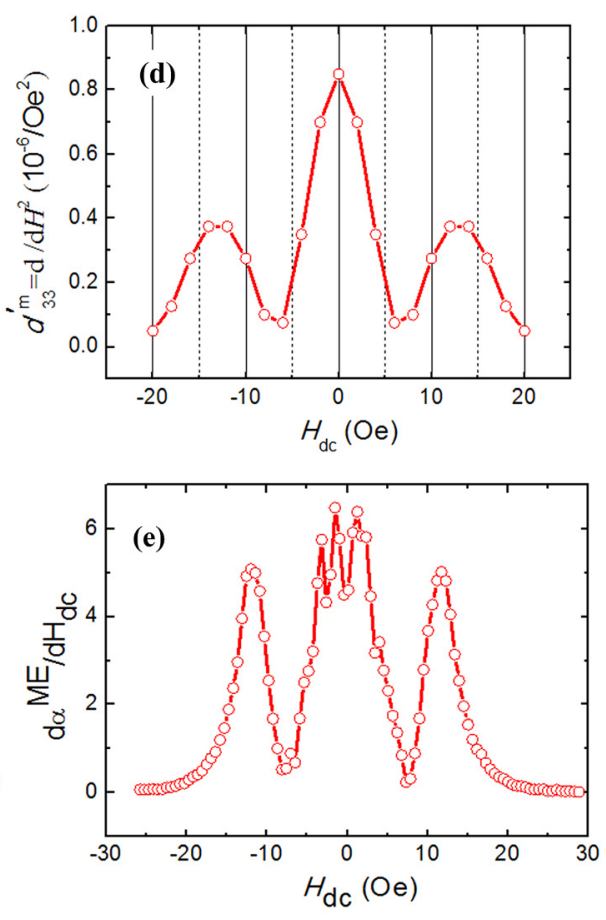

FIG. 2. (a) Non-linear ME voltage coefficient $\alpha_{\text {nonlin }}^{M E}$ of $8 \mathrm{~cm}$-long Metglas/ PMN-PT laminate composites projected on $H_{\mathrm{dc}}$. (b) Magnetostriction strain $\lambda$ (black curve) and piezomagnetic coefficient $d_{33}^{m}$ (blue curve) as a function of $H_{\mathrm{dc}}$. (c) The first order ME coefficient $\alpha^{\mathrm{ME}}$ curve which is shown to be highly dependent on the piezomagnetic coefficient $d_{33}^{m}$. (d) Derivative strength of $d_{33}^{m}$ as a function of $H_{\mathrm{dc}}$. (e) The derivative strength of ME coefficient $\alpha^{\mathrm{ME}}$. It indicates that $\alpha_{\text {nonlin }}^{M E}$ has a differential dependence of $d_{33}^{m}$ on magnetic bias $H_{\mathrm{dc}}$ that is $\partial d_{33}^{m} / \partial H$. 
magnetic field $H(t)=H_{d c}+H_{a c} \sin \left(2 \pi f_{0} t\right)+H_{\text {inc }} \sin \left(2 \pi f_{1} t\right)$ is induced. This induced ME output can be measured by an AC-coupling method, where the DC component of the signals is removed. Thus, the 2nd order cross-term $\alpha_{2}^{\prime} H_{\text {inc }} H_{a c} \cos \left[2 \pi\left(f_{0} \pm f_{1}\right) t\right]$ becomes significant, where the new summed parameter $\alpha_{2}^{\prime}$ equals $\alpha_{\text {nonlin }}^{\mathrm{ME}}$ based on its definition (see Eq. (1)). The Taylor series also has a definition for the 2 nd order coefficient $\alpha_{2}^{\prime}=\frac{d V^{2}}{d^{2} H}$ of

$$
\alpha_{\mathrm{nonlin}}^{\mathrm{ME}}=\alpha_{2}^{\prime}=\frac{d V^{2}}{d^{2} H}=\frac{d \alpha_{1}}{d H}=\frac{d \alpha^{M E}}{d H} .
$$

Equation (3) indicates that $\alpha_{\text {nonlin }}^{\mathrm{ME}}$ should be proportional to the derivative strength of $\alpha^{\mathrm{ME}}$, which agrees well with experiment results. Comparison of Figures 2(a) and 2(e) illustrates this. The values of $\alpha_{\text {nonlin }}^{\mathrm{ME}}$ and $\partial \alpha^{M E} / \partial H$ have similar functional forms with $H_{\mathrm{dc}}$ : both have a first peak around zero DC bias, decrease to a minimum at $H_{\mathrm{dc}}= \pm 9 \mathrm{Oe}$, and subsequently increase to a second peak near $H_{\mathrm{dc}}= \pm 13$ Oe. Since $\alpha^{\mathrm{ME}}$ is proportional to $d_{33}^{m}$ (see Fig. 2(b)), Eq. (3) reduces to

$$
\alpha_{\text {nonlin }}^{\mathrm{ME}} \propto \frac{\partial d_{33}^{m}}{\partial H} .
$$

This equation shows that $\alpha_{\text {nonlin }}^{\mathrm{ME}}$ should be proportional to $\partial d_{33}^{m} / \partial H$. Equation (4) is verified by the data in Figures 2(a) and $2(\mathrm{c})$, as again the functional forms of $\alpha_{\mathrm{nonlin}}^{\mathrm{ME}}$ and $\partial d_{33}^{m} / \partial H$ with $H_{\mathrm{dc}}$ are similar.

A magnetic flux concentration effect was then found to enhance $\alpha_{\text {nonlin }}^{\mathrm{ME}}$. Figure 3(a) shows the value of $\alpha_{\text {nonlin }}^{\mathrm{ME}}$ for laminates with different Metglas lengths $(\mathrm{L}=6 \mathrm{~cm}$ and $10 \mathrm{~cm})$ as a function of $H_{\mathrm{dc}}$. In this figure, it can be seen for $\mathrm{L}=6 \mathrm{~cm}$ that the peaks in $\alpha_{\text {nonlin }}^{\mathrm{ME}}$ became wider than the one with $8 \mathrm{~cm}$ Metglas foils (see Figure 2(a)); in particular, the 2nd peak was shifted to a higher bias of $H_{\mathrm{dc}}= \pm 20 \mathrm{Oe}$, and the minimum between the two peaks was increased to $H_{\mathrm{dc}}= \pm 12 \mathrm{Oe}$. It can also be seen that the peak values were significantly decreased. In contrast, for $\mathrm{L}=10 \mathrm{~cm}$, the peak values of $\alpha_{\text {nonlin }}^{\mathrm{ME}}$ were notably increased: the peak value was $9.5 \mathrm{~V} /\left(\mathrm{cm}-\mathrm{Oe}^{2}\right)$ at $H_{\mathrm{dc}}=-1$ Oe for the 1 st peak, which represents an enhancement of a factor of $4 \times$ for $\alpha_{\text {nonlin }}^{\mathrm{ME}}$ over previous reports using a $1 \mathrm{kHz}$ driving signal. ${ }^{12}$ It should also be noted under $H_{\mathrm{dc}}=0$ that the value of $\alpha_{\mathrm{nonlin}}^{\mathrm{ME}}$ was increased to $7.5 \mathrm{~V} /\left(\mathrm{cm}-\mathrm{Oe}^{2}\right)$ : this is in comparison to that of $2.5 \mathrm{~V} /\left(\mathrm{cm}-\mathrm{Oe}^{2}\right)$ for $\mathrm{L}=6 \mathrm{~cm}$ and $4.8 \mathrm{~V} /\left(\mathrm{cm}-\mathrm{Oe}^{2}\right)$ for $\mathrm{L}=8 \mathrm{~cm}$. In addition, for $\mathrm{L}=10 \mathrm{~cm}$, the peaks of $\alpha_{\text {nonlin }}^{\mathrm{ME}}$ with $H_{\mathrm{dc}}$ were sharper and occurred at lower biases. These trends of change in $\alpha_{\text {nonlin }}^{\mathrm{ME}}$ can be attributed to the difference in $d_{33}^{m}$ for different Metglas lengths, as shown in Figure 3(b). In this figure, for $\mathrm{L}=6 \mathrm{~cm}$, one can see that the maximum $d_{33}^{m}$ occurred near $H_{\mathrm{dc}}= \pm 12 \mathrm{Oe}$, whereas for $\mathrm{L}=10 \mathrm{~cm}$, the $d_{33}^{m}$ peak value was shifted to $H_{\mathrm{dc}}= \pm 6$ Oe. This resulted in an increased slope of $\mathrm{d}_{33}^{\mathrm{m}}$ with $H_{\mathrm{dc}}$, especially near $H_{\mathrm{dc}}=0$ as highlighted in the figure. These results demonstrate that $\alpha_{n o n l i n}^{M E}$ is notably dependent on $\partial d_{33}^{m} / \partial H$ and that the values of both can be increased by flux concentration.

When the cross-modulation signal output was demodulated and multiplied with the driving signal $H_{a c} \sin \left(2 \pi f_{0} t\right)$, the original incident signal $f_{1}$ can be recovered in the term
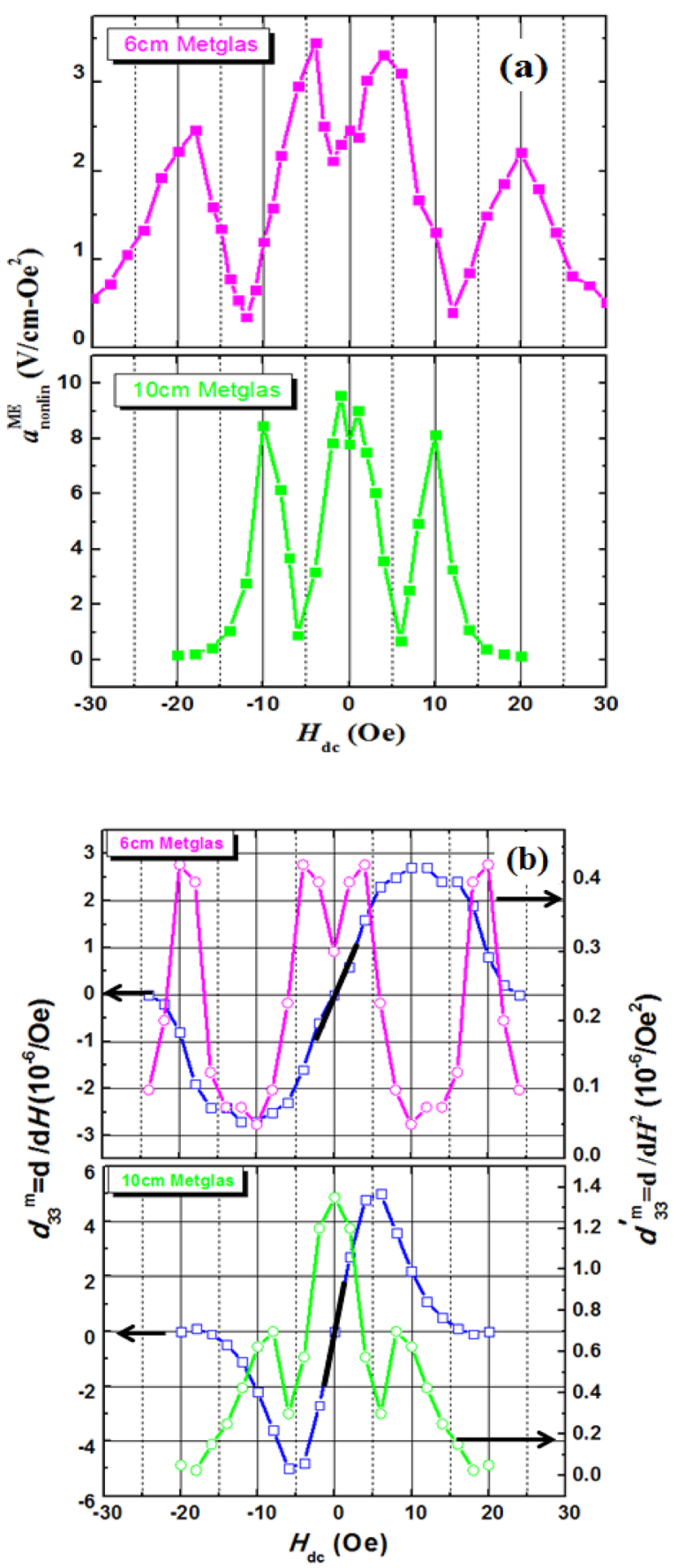

FIG. 3. (a) ME non-linear coefficients $\alpha_{\text {nonlin }}^{M E}$ for different lengths $(6 \mathrm{~cm}$ and $10 \mathrm{~cm}$ ) Metglas as a function of $H_{\mathrm{dc}}$. (b) Piezomagnetic coefficient $d_{33}^{m}$ and its derivative strength $\partial d_{33}^{m} / \partial H$ of $6 \mathrm{~cm}$ and $10 \mathrm{~cm}$ composites. The $10 \mathrm{~cm}$ composites have much higher $\partial d_{33}^{m} / \partial H$ value than that of $6 \mathrm{~cm}$ one, particularly under $H_{\mathrm{dc}}=0$ Oe. The slope of $\mathrm{d}_{33}^{\mathrm{m}}$ is highlighted by a bold black line.

$H_{\text {inc }} H_{a c}^{2} \cos \left(2 \pi f_{1}\right) t$ (see dashed window in Fig. 1). ${ }^{15}$ Following the modulation principle, the demodulation voltage output $V_{\mathrm{de}}$ should be linear proportional to the incident field $H_{\text {inc. }}$. Accordingly, we measured the sensor output signal after demodulation as a function of $H_{\text {inc }}$ using the $8 \mathrm{~cm}$ ME laminates as given in Figure 4(a). Laminates of length $6 \mathrm{~cm}$ and $10 \mathrm{~cm}$ have similar results that are not shown here. The carrier signal $(1 \mathrm{kHz})$, two cross-modulation signals $(999$ and $1001 \mathrm{~Hz})$, and the demodulated signal $(1 \mathrm{~Hz})$ can be identified. In this figure, it can also be seen as $H_{\text {inc }}$ was increased from $18 \mathrm{nT}$ to $180 \mathrm{nT}$ that enhanced crossmodulation signals resulted, which in turn lead to an 

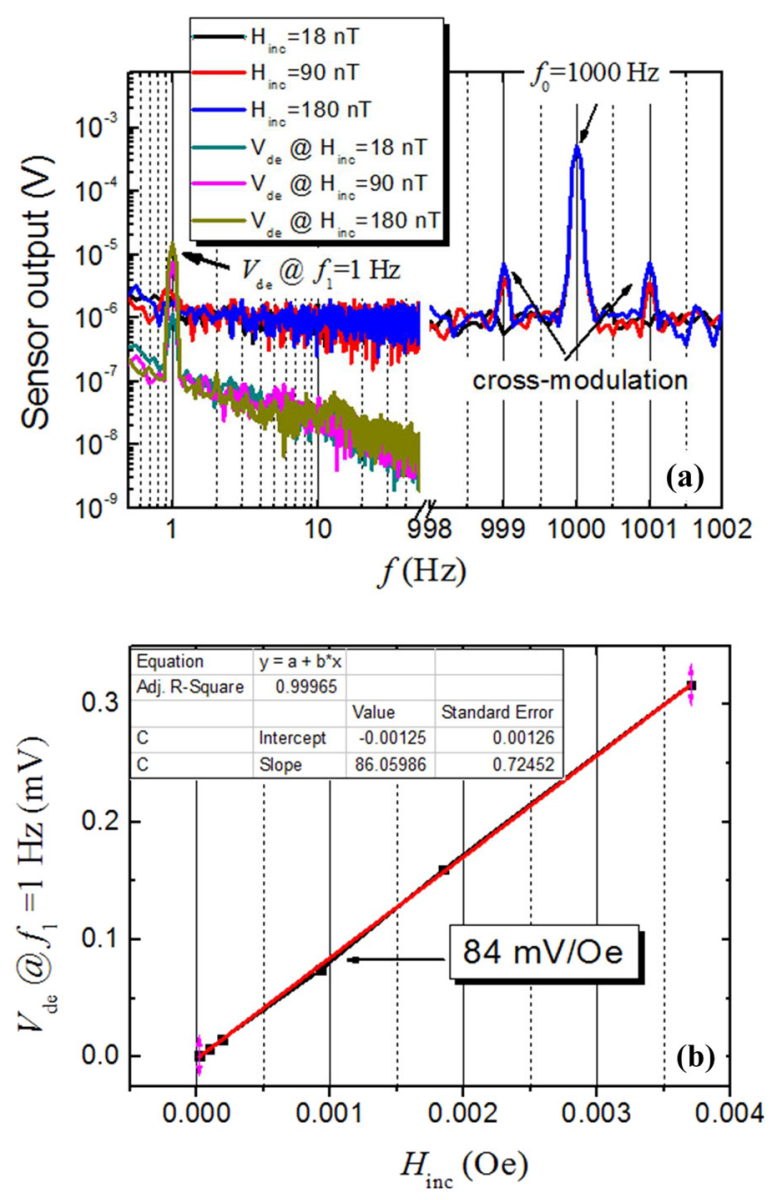

FIG. 4. (a) Sensor output signals after demodulation as a function of $H_{\text {inc }}$ using the $8 \mathrm{~cm}$ ME composites which contain the carrier signal $(1 \mathrm{kHz})$, two crossmodulation signals $(999$ and $1001 \mathrm{~Hz}$ ), and the demodulated signal $(1 \mathrm{~Hz})$. The increased $H_{\text {inc }}$ from $1.8 \mathrm{nT}$ to $18 \mathrm{nT}$ results in enhanced cross-modulation signals and finally leads to an increased demodulated signal $V_{\mathrm{de}}$ at $f_{1}=1 \mathrm{~Hz}$. (b) Demodulation output $V_{\mathrm{de}}$ is strongly dependent on $H_{\text {inc }}$ with demodulation transfer ability function of $84 \mathrm{mV} / \mathrm{Oe}$ when driving signal $H_{\mathrm{ac}}=0.074 \mathrm{Oe}$.

increased demodulated signal at $f_{1}=1 \mathrm{~Hz}$. In Figure 4(b), the value of $V_{\mathrm{de}}$ can be seen to be dependent on $H_{\text {inc }}$ with a demodulation transfer function equal to $84 \mathrm{mV} / \mathrm{Oe}$. Note that these data were measured at a constant driving signal of $H_{\mathrm{ac}}=0.074 \mathrm{Oe}$ for $f_{0}=1 \mathrm{kHz}$ under zero DC bias. The results show that $V_{\mathrm{de}}$ is linearly proportional to $H_{\text {inc }}$, in agreement with the modulation principle. Such a linearity of the transfer function is essential for estimating unknown incident fields in applications, where normalization is difficult to perform accurately.
In summary, the non-linear ME coefficient $\alpha_{\text {nonlin }}^{\mathrm{ME}}$ for ME laminate composites of Metglas/PMN-PT has been found to be strongly dependent on $\partial d_{33}^{m} / \partial H$. Adjusting the slope of $d_{33}^{m}$ by a flux-concentration effect provides a way to improve $\alpha_{n o n l i n}^{M E}$ at $H_{\mathrm{dc}}=0 \mathrm{Oe}$. This is important to remove the required $H_{\mathrm{dc}}$ for ME sensors, eliminating potential bias interference effects in sensor arrays. The results demonstrate the feasibility to also use the modulation technique to reduce vibration noise by shifting low frequency magnetic signal $\left(f_{1}\right)$ to higher frequencies $\left(f_{0} \pm f_{1}\right)$. By multiplying crossmodulation signals with the driving signal, an incident signal $\left(f_{1}\right)$ was recovered filtering out low frequency noises. The demodulated signal $V_{\text {de }}$ was found to be linearly proportional to $H_{\text {inc }}$, in agreement with predictions. An increase of the Metglas length resulted in increased values of $\alpha_{\text {nonlin }}^{\mathrm{ME}}$ to $7.5 \mathrm{~V} /\left(\mathrm{cm}-\mathrm{Oe}^{2}\right)$ and to $9.5 \mathrm{~V} /\left(\mathrm{cm}-\mathrm{Oe}^{2}\right)$ at $H_{\mathrm{dc}}=0$ and -1 Oe, respectively.

The authors thank the Office of Naval Research for funding.

${ }^{1}$ J. V. D. Boomgaard, A. M. J. G. V. Run, and J. V. Suchtelen, Ferroelectrics 10, 295 (1976).

${ }^{2}$ W. Eerenstein, N. D. Mathur, and J. F. Scott, Nature (London) 442(7104), 759-765 (2006).

${ }^{3}$ C.-W. Nan, M. I. Bichurin, S. Dong, D. Viehland, and G. Srinivasan, J. Appl. Phys. 103(3), 031101 (2008).

${ }^{4}$ G. Srinivasan, E. Rasmussen, J. Gallegos, R. Srinivasan, Y. Bokhan, and V. Laletin, Phys. Rev. B 64(21), 214408 (2001).

${ }^{5}$ J. Ma, J. Hu, Z. Li, and C.-W. Nan, Adv. Mater. 23(9), 1062-1087 (2011).

${ }^{6}$ Y. Shen, J. Gao, Y. Wang, J. Li, and D. Viehland, Appl. Phys. Lett. 100(17), 173505 (2012).

${ }^{7}$ Y. Shen, J. Gao, D. Hasanyan, Y. Wang, M. Li, J. Li, and D. Viehland, Smart Mater. Struct. 21(11), 115007 (2012).

${ }^{8}$ J. Gao, Y. Shen, P. Finkel, J. Blottman, J. Li, and D. Viehland, Mater. Lett. 88, 47-50 (2012).

${ }^{9}$ J. Gao, Z. Wang, Y. Shen, M. Li, Y. Wang, P. Finkel, J. Li, and D. Viehland, Mater. Lett. 82, 178-180 (2012).

${ }^{10}$ Y. Wang, J. Gao, M. Li, D. Hasanyan, Y. Shen, J. Li, D. Viehland, and H. Luo, Appl. Phys. Lett. 101(2), 022903 (2012).

${ }^{11}$ T.-Z. Wang and Y.-H. Zhou, Compos. Struct. 93(5), 1485-1492 (2011).

${ }^{12}$ L. Shen, M. Li, J. Gao, Y. Shen, J. F. Li, D. Viehland, X. Zhuang, M. L. C. Sing, C. Cordier, S. Saez, and C. Dolabdjian, J. Appl. Phys. 110(11), 114510 (2011).

${ }^{13}$ S. M. Gillette, A. L. Geiler, D. Gray, D. Viehland, C. Vittoria, and V. G. Harris, IEEE Magn. Lett. 2, 2500104 (2011).

${ }^{14}$ S. Dong, J. Zhai, J. Li, and D. Viehland, Appl. Phys. Lett. 89(25), 252904 (2006).

${ }^{15}$ X. Zhuang, M. L. C. Sing, C. Cordier, S. Saez, C. Dolabdjian, L. Shen, J. F. Li, M. Li, and D. Viehland, IEEE Sens. J. 11(10), 2266-2272 (2011). 